\title{
When are active Brownian particles and run-and-tumble particles equivalent? Consequences for motility-induced phase separation
}

\author{
M. E. Cates $^{1}$, J. Tailleur ${ }^{2}$ \\ 1 SUPA, School of Physics, University of Edinburgh, JCMB Kings Buildings, Edinburgh EH9 3JZ, United Kingdom \\ 2 Univ Paris Diderot, Sorbonne Paris Cité, MSC, UMR 7057 CNRS, F75205 Paris, France
}

PACS 05.40.-a - Fluctuation phenomena: statistical physics

PACS 87.10.Mn - Stochastic models in biological physics

PACS $64.75 . \mathrm{Jk}$ - phase separation and segregation in Nanoscale systems

\begin{abstract}
Active Brownian particles (ABPs, such as self-phoretic colloids) swim at fixed speed $v$ along a body-axis $\mathbf{u}$ that rotates by slow angular diffusion. Run-and-tumble particles (RTPs, such as motile bacteria) swim with constant $\mathbf{u}$ until a random tumble event suddenly decorrelates the orientation. We show that when the motility parameters depend on density $\rho$ but not on $\mathbf{u}$, the coarse-grained fluctuating hydrodynamics of interacting ABPs and RTPs can be mapped onto each other and are thus strictly equivalent. In both cases, a steeply enough decreasing $v(\rho)$ causes phase separation in dimensions $d=2,3$, even when no attractive forces act between the particles. This points to a generic role for motility-induced phase separation in active matter. However, we show that the ABP/RTP equivalence does not automatically extend to the more general case of $\mathbf{u}$-dependent motilities.
\end{abstract}

The physics of self-propelled colloidal particles represents a central focus of research into 'active matter' [1-3]. In active matter, a continuous supply of energy destroys microscopic time-reversibility and allows phenomena to arise that are impossible in thermal equilibrium systems, where detailed balance restores time-reversal symmetry in the steady state.

One class of self propelled colloids is represented by motile bacteria such as Escherichia coli, which move in a sequence of 'runs' - periods of almost straight-line motion at near-constant speed $v$-punctuated by sudden and rapid reassignments of direction, or 'tumbles', occurring at random with rate $\alpha$ 4, 5. (Certain algae have similar motion [6].) At large scales, and under conditions of constant $v, \alpha$, a single run-and-tumble particle (RTP) performs a random-walk of diffusivity $D_{0}=v^{2} / \alpha d(d$ is dimensionality) which cannot be distinguished from the equilibrium dynamics of non-interacting passive Brownian particles (PBPs). Nonetheless, it was shown previously that if their swim speed $v(\rho)$ decreases fast enough with the local particle density $\rho$, RTPs undergo phase separation between a dense, slow-swimming fluid and a dilute fast-swimming one 7]. This happens even in the complete absence of interparticle attractive forces, which are a prerequisite for fluid-fluid phase separation in thermal
PBPs. Coupled to population dynamics, this motilityinduced phase separation was implicated in the patterning of growing bacterial colonies 8 10.

A second class of self-propelled colloids are represented by synthetic (bi-)metallised colloids which, usually by catalysing the breakdown of hydrogen peroxide, create a self-phoretic local chemical motor [11-15]. These differ from RTPs in that the direction of swimming only changes gradually, by rotational diffusion (with angular diffusivity $\left.D_{r}\right)$. This rotation is usually Brownian in origin, hence the name ABPs (active Brownian particles). Biological ABPs also exist, including non-tumbling $E$ coli mutants and other organisms that self-propel without tumbling. Clearly an isolated ABP again performs a random walk $\left(D_{0} \propto v^{2} / D_{r}\right)$ at large scales.

When all microscopic parameters are uniform and isotropic, the large scale dynamics of RTPs, ABPs and PBPs are thus described by the diffusion equation. Equating their diffusivities thus trivially makes their dynamics identical. In the converse case, for instance when interactions, external potentials, or spatially varying swimming parameters are present, the dynamics become more complex and typically involve a drift term:

$$
\dot{\rho}=-\nabla \cdot\left[\mathbf{V}\left(v, \alpha, D_{r}, \ldots\right) \rho-D\left(v, \alpha, D_{r}, \ldots\right) \nabla \rho\right]
$$


While the microscopic parameters can clearly still be chosen so that the diffusivities of the three processes are equal, there is no reason why the equality of the drift terms $\mathbf{V}$ should then follow as a by-product. Following Schnitzer [4, this point can be understood by considering PBPs in which either viscosity or temperature are nonuniform in space, both leading to the same nonuniform $D(\mathbf{r})$. As required by detailed balance for an isothermal system, $\mathbf{V}$ is zero in the first case despite the nonuniform diffusivity. But if the same diffusivity variation arises instead through a non-uniform $T(\mathbf{r})$, detailed balance is broken, $\mathbf{V}$ is nonzero, and no generic Einstein-like relation relates $D$ to $\mathbf{V}$. Likewise, because ABPs and RTPs are out of equilibrium, the mere existence of a diffusive behaviour in the uniform case does not establish the equivalence of ABPs with either PBPs or RTPs in more general situations. It is therefore intriguing that recent simulations of spherical ABPs with purely repulsive interactions showed clear signs of phase separation 16, 17, something very similar to what is observed for RTPs 18].

By analogy with what we know for RTPs, such a phase separation is explicable if the repulsions act to effectively decrease $v$ at high density (by caging, for instance). However, the growing interest in ABPs [3] (exemplified by several other recent theory/simulation studies and continuing experiments 19 26]) clearly requires a treatment of this subject that lies beyond mere analogy. Are ABPs and RTPs actually equivalent, and if so under what conditions?

This Letter provides a partial answer to that important question. At time scales large compared to the angular reorientation time $\tau\left(\sim 1 / D_{r}\right.$ or $\left.\sim 1 / \alpha\right)$, and length scales large compared to $\ell=v \tau$, we establish a leading-order exact equivalence between ABPs and RTPs so long as the motility parameters $v, \alpha, D_{r}$ are independent of particle orientation $\mathbf{u}$. This result, which extends to the case where a Brownian translational diffusivity $D_{t}$ is added, also holds when these parameters depend (slowly) on position. Following [7], this allows treatment of interacting particles, as long as the effect of interactions can be faithfully described by density-dependent swimming parameters. This can for instance be the case when interactions are mediated by rapidly diffusing chemicals $[9,10$ or when mean-field treatments are valid (either as a result of molecular chaos or when interactions are averaged over many neighbouring particles as in $[18$ ). Thus the ABP-RTP equivalence indeed holds under the conditions (generalised here to $d>1$ ) shown in 7 to cause motility-induced bulk phase separation in RTPs. Accordingly ABPs will generically show the same sort of phase separation, subject to the same requirement of a sufficiently decreasing $v(\rho)$.

Because we assume slow variations of all quantities on the scale of $\ell$, we cannot rule out differences between ABPs and RTPs in situations involving ordering at shorter scales, such as the solidification transition addressed in 23]. Moreover, as we prove later on, no general equivalence exists for cases involving $\mathbf{u}$-dependent motility parameters. (This shows that while some degree of simi- larity between ABPs and RTPs might be expected, any actual equivalence between them is not automatic.) Instances of such anisotropic dynamics include bacterial rectification 24,27, as modelled by an orientation-dependent tumble rate 2, 28, sedimentation, where the particle speed acquires a gravitational component $14,22,28$ and systems of ABPs in which interactions promote alignment $3,21,29,33$. Furthermore, hydrodynamic interactions, neglected entirely here, have subtle consequences 3438 that might well differ between RTPs and ABPs. Despite these caveats, it is interesting to establish an asymptotic equivalence between ABPs and RTPs in a sector of parameter space that includes a nontrivial manybody phenomenon, namely motility-induced bulk phase separation.

Fluctuating hydrodynamics. We start by analysing a single particle undergoing a generalized run-and-tumble dynamics with both rotational and translational diffusions, and later recover RTP and ABP as limiting cases. We allow, $v, \alpha, D_{r}$ and $D_{t}$ to depend on position but not orientation. The probability density $\psi(\mathbf{r}, \mathbf{u}, t)$ of finding a particle at position $\mathbf{r}$ moving in direction $\mathbf{u}$ obeys exactly (in $d=2,3$ )

$$
\begin{aligned}
& \dot{\psi}(\mathbf{r}, \mathbf{u})=-\nabla \cdot[v \mathbf{u} \psi(\mathbf{r}, \mathbf{u})]+\nabla_{\mathbf{u}}\left[D_{r} \nabla_{\mathbf{u}} \psi(\mathbf{r}, \mathbf{u})\right] \\
& \quad+\nabla\left(D_{t} \nabla \psi(\mathbf{r}, \mathbf{u})\right)-\alpha \psi(\mathbf{r}, \mathbf{u})+\frac{\alpha}{\Omega} \int \psi\left(\mathbf{r}, \mathbf{u}^{\prime}\right) \mathrm{d} \Omega^{\prime}
\end{aligned}
$$

where $\nabla_{\mathbf{u}}$ is the rotational gradient acting on $\mathbf{u}$ and the integral is over the unit sphere $\left|\mathbf{u}^{\prime}\right|=\mathbf{1}$ of area $\Omega$. The first term on the right is the divergence of the advective current resulting from self propulsion and the last two terms are loss and gain terms due to tumbling in and out of the direction $\mathbf{u}$. We next decompose $\psi$ as

$$
\psi(\mathbf{r}, \mathbf{u}, t)=\varphi+\mathbf{p . u}+\mathbf{Q}:(\mathbf{u u}-\mathbf{I} / d)+\Theta[\psi]
$$

Here, $\varphi, \mathbf{p}, \mathbf{Q}$ are functions of $(\mathbf{r}, t)$ but not $\mathbf{u}$, which parameterize the zeroth, first and second angular $(d=2)$ or spherical $(d=3)$ harmonic components of $\psi$, while $\Theta$ projects onto the higher harmonic components 39 .

Integrating eq. (2) over $\mathbf{u}$ gives

$$
\dot{\varphi}=-\frac{1}{d} \nabla(v \mathbf{p})+\nabla\left(D_{t} \nabla \varphi\right)
$$

Using the orthogonality of angular/spherical harmonics, one can then obtain equations order by order. Multiplying eq. (2) by $\mathbf{u}$ and integrating over $\mathbf{u}$ then yields, after some algebra [40], for each component of $\mathbf{p}$

$$
\begin{aligned}
\dot{p}_{a} & =-\nabla_{a}(v \varphi)-\left(D_{r}(d-1)+\alpha\right) p_{a}+\nabla\left(D_{t} \nabla p_{a}\right) \\
& -B_{a b c d} \nabla_{b}\left(v Q_{c d}\right)
\end{aligned}
$$

where $B_{a b c d}=\left(\delta_{a c} \delta_{b d}+\delta_{a d} \delta_{b c}-2 \delta_{a b} \delta_{c d} / d\right) /(d+2)$.

Similarly, multiplying eq. (2) by $\mathbf{u u}-\mathbf{I} / d$ and integrating over $\mathbf{u}$ yields (with $\chi_{a b c}$ defined below)

$$
\begin{aligned}
\dot{Q}_{a b} & =-\frac{d+2}{2} B_{a b c d} \nabla_{c}\left(v p_{d}\right)-\nabla_{c}\left(\chi_{a b c}\right) \\
& -\left(2 d D_{r}+\alpha\right) Q_{a b}+\nabla\left(D_{t} \nabla Q_{a b}\right)
\end{aligned}
$$


Equation (4) states that the rate of change of the probability density of finding the particle at position $\mathbf{r}$ irrespectively of its orientation (zeroth harmonic, $\varphi$ ) is the negative divergence of a flux $\mathbf{J}=v \mathbf{p} / d-D_{t} \nabla \varphi$ which depends solely on the two first harmonics. In addition to the diffusive flux $-D_{t} \nabla \varphi$, there is a contribution of the self-propulsion along $\mathbf{p}$.

In eq. (5), one sees that $\dot{\mathbf{p}}$ relaxes via a flux term but also directly by angular diffusion and tumbling: here $(1-d)$ is the eigenvalue of $\Delta_{\mathbf{u}}$ for the first harmonic (likewise $-2 d$ for the second in eq. (6)). The last term gives the contribution to $\dot{\mathbf{p}}$ arising from the $\mathbf{Q}$ term within $\nabla .[v \mathbf{u} \psi]$ in eq. $[2]$. There are no contributions to $\dot{\mathbf{p}}$ from the higher harmonics but there is one from the zeroth harmonic: the $\nabla_{a}(v \varphi)$ term. It encodes the fact that, although an isotropic distribution of swimmers cannot transport density if $D_{t}=0$, it can still create anisotropy; for instance if the swim speed or density is higher to the right of the origin, an initially isotropic density at the origin soon develops an excess of left-moving swimmers.

A corresponding set of remarks apply to eq. (6) for the time evolution of $\mathbf{Q}$. Here $B_{a b c d} \nabla_{c}\left(v p_{d}\right)$ is a flux contribution from the first harmonic whereas $\chi_{a b c}$ (whose form we don't need) arises from the higher harmonics in $\Theta[\psi]$. Finally, the evolution of all remaining harmonics is obtained, if needed, by projecting eq. (2) using $\Theta$.

So far, beyond the assumed isotropy of $v(\mathbf{r}), D_{r, t}(\mathbf{r})$ and $\alpha(\mathbf{r})$, no approximation has been made; eqs. (4,6) are exact results for the time evolution of the zeroth, first and second harmonics of $\psi(\mathbf{r}, \mathbf{u}, t)$. At this stage, despite similar structures, the dynamics of ABP and RTP cannot be mapped onto each other. Equating the prefactors of $p_{a}$ in (5) indeed requires $(d-1) D_{r}=\alpha$ which makes the prefactors of $Q_{a b}$ in $(6)$ unequal since then $2 d D_{r} \neq \alpha$.

We now wish to coarse-grain these equations to obtain a 'diffusion/drift' expression for the flux $\mathbf{J}$ that involves only the conserved probability density $\varphi(\mathbf{r}, t)$, the slowly varying parameters $D_{r, t}, v, \alpha$, and their first derivatives:

$$
\dot{\varphi}=-\nabla . \mathbf{J} \quad ; \quad \mathbf{J}=\mathbf{V}(\mathbf{r}, t) \varphi-D(\mathbf{r}, t) \nabla \varphi
$$

Following 7 , we use a gradient expansion and first note that $\varphi$ is the only slow mode: its relaxation time is of order $\sim(\nabla)^{-1}$ whereas all other harmonics relax in times of order $\sim 1$. We thus assume $\dot{\mathbf{p}}=\dot{\mathbf{Q}}=\Theta[\dot{\psi}]=0$ when calculating the quasi-stationary current $\mathbf{J}$. By itself, this creates a non-local constitutive relation between $\mathbf{J}$ and $\psi$ which still involves all harmonics. Next we carry out explicitly the gradient expansion, yielding for $\mathbf{Q}$ :

$$
Q_{i j}=-\frac{\frac{d+2}{2} B_{i j k \ell} \nabla_{k}\left(v p_{\ell}\right)+\nabla_{k} \chi_{i j k}}{2 d D_{r}+\alpha}+\mathcal{O}\left(\nabla^{2}\right)
$$

from which the quasi-stationary $\mathbf{p}$ then follows via (5) as

$$
\mathbf{p}=-\frac{1}{(d-1) D_{r}+\alpha} \nabla(v \varphi)+O\left(\nabla^{2}\right)
$$

Fortunately therefore, to diffusion-drift order, closure is achieved without needing further information on harmonics beyond the first. To this order eq. (7) holds, with

$$
\mathbf{J}=-\frac{v}{d(d-1) D_{r}+d \alpha} \nabla(v \varphi)-D_{t} \nabla \varphi
$$

so that the diffusivity and drift velocity obey

$$
D=\frac{v^{2}}{d(d-1) D_{r}+d \alpha}+D_{t} ; \mathbf{V}=\frac{-v \nabla v}{d(d-1) D_{r}+d \alpha}
$$

Eqs. (7) and (10) give the evolution for the probability density of one particle at diffusion-drift level. They are equivalent to an Ito-Langevin equation for an individual particle position $\mathbf{r}_{\mu}(t)$. Following 7 ] we can therefore now consider an assembly of particles whose motility parameters $v, \alpha, D_{r}$ and $D_{t}$ depend on position through a set of (smooth) density functionals. The coarse-grained density $\rho(\mathbf{r}, t)$ on which these depend then obeys the many body Langevin equation 7

$$
\dot{\rho}=-\nabla \cdot\left(\mathbf{V}[\rho] \rho-D[\rho] \nabla \rho+(2 D \rho)^{1 / 2} \boldsymbol{\Lambda}\right)
$$

with white noise $\left\langle\Lambda_{i}(\mathbf{r}, t) \Lambda_{j}\left(\mathbf{r}^{\prime}, t^{\prime}\right)\right\rangle=\delta_{i j} \delta\left(\mathbf{r}-\mathbf{r}^{\prime}\right) \delta\left(t-t^{\prime}\right)$. This noise term serves as a reminder that $\rho(\mathbf{r}, t)$ is not the one-particle probability $\varphi(\mathbf{r}, t)$ but a smooth coarsegraining of the collective density field $\sum_{\mu} \delta\left(\mathbf{r}-\mathbf{r}_{\mu}(t)\right.$ ) (with $\mu$ a particle index) which evolves stochastically. The functionals $v[\rho], \alpha[\rho]$ and $D_{t, r}[\rho]$ in 10$]$ then defines for the interacting particle system the many-body drift velocity and diffusivity $\mathbf{V}[\rho]$ and $D[\rho]$ for use in eq. (11).

We can now use eqs. (1011) to compare the dynamics of RTPs and ABPs. From the expression of $D$ and $\mathbf{V}$ in (10), we see that the tumble rate $\alpha$ and rotational diffusivity $D_{t}$ enter only through the combination $(d-1) D_{r}+\alpha \equiv$ $\tilde{\alpha}$. Hence the pure RTP and pure ABP limits, alongside anything in between, are made equivalent by a suitable choice of $\tilde{\alpha}[\rho]$. The effect of nonzero $D_{t}$ is the same in each case, although we note that, in many experimental cases, the self-propelling speed $v$ is large enough that the translational diffusivity $D_{t}$ is negligible. (For instance in wild-type run-and-tumble bacteria, $v^{2} /(d \alpha)$ is about three orders of magnitude larger than $D_{t}$.) In this limit, pure $\operatorname{ABPs}\left(D_{t}=\alpha=0\right)$ and pure RTPs $\left(D_{t}=D_{r}=0\right)$ are equivalent up to the mapping $(d-1) D_{r} \leftrightarrow \alpha$.

Motility-induced phase separation. The manybody physics predicted by eqs. 10 111) for the large class of active particle systems addressed above essentially coincides with that studied in 7 for RTPs in $d=1$. (Note that ABPs cannot be defined in $d=1$.) As shown there, because (11) is equivalent to a set of interacting PBPs with the specified diffusivity and drift functionals, a condition can be found under which the interacting active system will behave just like a fluid of PBPs with free energy (in thermal units) $\mathcal{F}[\rho]=\mathcal{F}_{e x}[\rho]+\int \rho(\ln \rho-1) d x$. The required condition is 7

$$
\mathbf{V}([\rho], \mathbf{r}) / D([\rho], \mathbf{r})=-\nabla\left(\delta \mathcal{F}_{e x}[\rho] / \delta \rho(\mathbf{r})\right)
$$


where the right hand side represents the force (i.e., excess chemical potential gradient) on a particle at $\mathbf{r}$.

In the case where $D_{t}=0$, the left hand side of (12) is simply $-\nabla \ln v[\rho]$ and we then require $\delta \mathcal{F}_{e x}[\rho] / \delta \rho(\mathbf{r})=$ $\ln (v([\rho] ; \mathbf{r})$. When the swim speed depends only locally on density, so that $v([\rho] ; \mathbf{r})=v(\rho(\mathbf{r}))$, we have $\mathcal{F}_{\text {ex }}=$ $\int f_{e x}(\rho(\mathbf{r})) d \mathbf{r}$ where $f_{e x}=\int_{0}^{\rho} \ln v(s) d s$. (Note that when $\tilde{\alpha} d D_{t}$ is a nonzero constant, this result generalizes to $f_{e x}=\frac{1}{2} \int_{0}^{\rho} \ln \left[v(s)^{2}+\tilde{\alpha} d D_{t}\right] d s$.) This form of excess free energy leads at mean-field level to a spinodal instability whenever $d v / d \rho<-v / \rho$, and to binodal conditions for phase coexistence given by the usual common tangent construction on $f=f_{e x}+\rho(\ln \rho-1)$ 7. Beyond mean field, the steady state probability for density fluctuations is governed by the 'Boltzmann-like' distribution $\exp [-\mathcal{F}]$ and these fluctuations will eventually cause phase separation at all densities between the binodals.

To understand the phase-separation dynamics in detail, to address the interfacial tension between phases, and also to confirm that the phase separation is equilibrium-like (rather than having, say, an ever-moving interface between the phases), would require a detailed examination of gradient terms lying beyond the present study. The same was initially true of the analysis of RTPs made in [7], but the relevant gradient terms were later identified explicitly 18. However, some of the gradient terms neglected via eq. (8) could in principle differ between ABPs and RTPs, even if these share the same functionals $\mathbf{V}[\rho]$ and $D[\rho]$. Accordingly, the somewhat technical exploration of these higher gradient terms is, for the $\mathrm{ABP}$ case, deferred to future work.

The similarity between RTPs and ABPs undergoing phase separation can nevertheless be explored numerically by comparing simulations of large populations of interacting ABPs and RTPs. To do so we set $D_{t}=0$ and consider a swimming speed $v[\rho(x)]=v_{0} \exp [-\lambda \phi \arctan (\rho / \phi)]$ which decreases exponentially at low density before saturating at finite but non-zero swimming speed ${ }^{1}$ for larger $\rho$. Numerically, $\rho(\mathbf{r})$ is computed by convoluting the number density $\sum_{\mu} \delta\left(\mathbf{r}-\mathbf{r}_{\mu}\right)$ with the function $f(\mathbf{r})=$ $Z \exp \left[-1 /\left(1-r^{2} / w^{2}\right)\right]$ with $Z$ a normalisation constant. For such a $v[\rho(x)]$, the free energy is bimodal as soon as $\lambda \phi>2$. Starting from identical uniform initial condition, the spinodal decomposition of 6400 RTPs and ABPs is shown in figure 1 and is consistent with the aforementioned mapping between ABPs and RTPs.

We can now turn back to the simulations of 16,17 which show that excluded volume interactions promote phase separation of ABPs. This result, like that found numerically for RTPs on a lattice [18, fits well with the scenario presented here, even though the interactions in 16, 17 are collisional rather than via a smooth density functional. Notably, the collisions considered in 16, 17 do not directly affect the orientation $\mathbf{u}$; at high density a

\footnotetext{
${ }^{1}$ As in 7 , the density in the dense phase diverges in the absence of excluded volume interactions if $v(\rho)$ vanishes at large $\rho$.
}
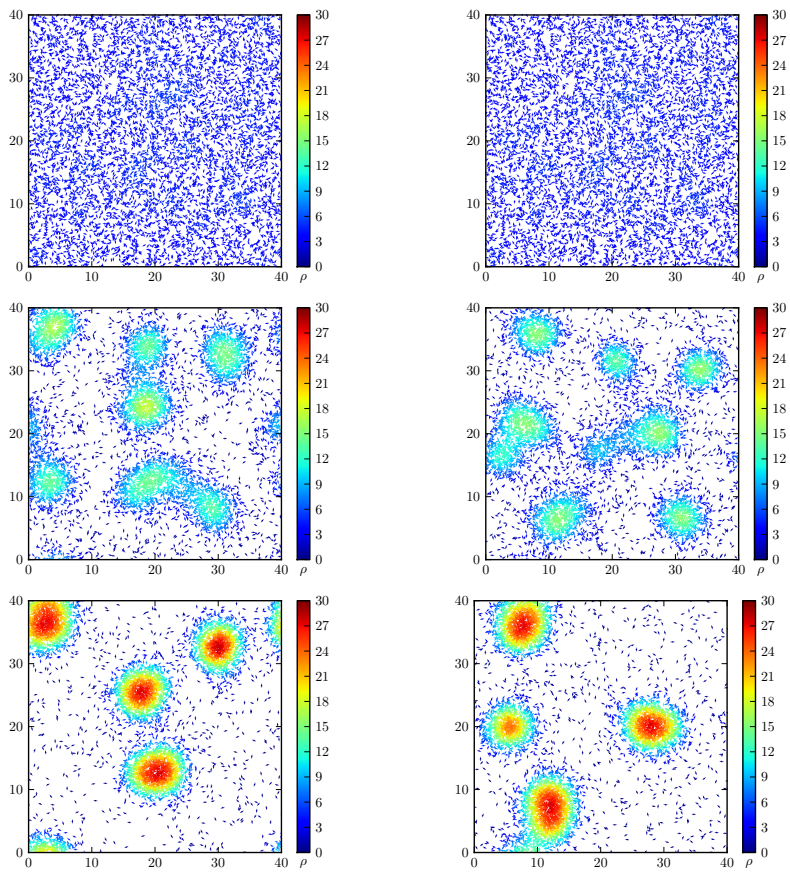

Fig. 1: Spinodal decomposition of $6400 \mathrm{ABP}$ (left) and RTP (right) in $2 \mathrm{D}$ with $v_{0}=10, \alpha=D_{r}=1.25, \lambda=0.45, \phi=7$, $w=3.5$ at times 0 (top), 100 (middle) and 1000 (bottom). Each particle appears as an arrow pointing along its body axis and with a colorcode equal to the local density $\rho(\mathbf{r})$.

particle undergoes many collisions before one-body rotational diffusion relaxes its swimming direction. Modelling collisions via $v(\rho)$ seems reasonable in this case. Indeed, $v(\rho)$ was reported in 16 to decay linearly, a case shown in [18] to cause phase separation for RTPs. Although a microscopic model starting directly with an effective $v(\rho)$ is only a mean-field-like description of the ABP simulations of 16, 17, the mechanistic similarity between their phase separation and that of RTPs is now clear.

Anisotropic motilities. We now turn to more general cases where the dynamics is not isotropic and discuss the limits of the equivalence between ABPs and RTPs. There are many ways of breaking isotropy and, as we show below, the mapping between RTPs and ABPs can be violated qualitatively, quantitatively, or preserved, depending on details of the microscopic dynamics.

One simple way to remove isotropy is to add a uniform field (such as gravity) that superimposes a Stokes drift velocity $\mathbf{w}$ on the self-propulsion. The overall velocity of a particle is then $v \mathbf{u}+\mathbf{w}$. This only affects the advection term in (2) and adds $-\nabla(\mathbf{w} \varphi),-\nabla_{b}\left(w_{b} p_{a}\right)$ and $-\nabla_{c}\left(w_{c} Q_{a b}\right)$ to the right hand side of equations (4 6). The large time/scale expansion then yields $Q \sim \nabla$ as before 
and one gets for $p$ and $\varphi$

$$
\begin{aligned}
\dot{\varphi} & =-\frac{1}{d} \nabla(v \mathbf{p})-\nabla(\mathbf{w} \varphi)+\nabla\left(D_{t} \nabla \varphi\right) \\
\left(D_{r}(d-1)+\alpha\right) p_{a} & =-\nabla_{a}(v \varphi)-\nabla_{b}\left(w_{b} p_{a}\right)+\mathcal{O}\left(\nabla^{2}\right)
\end{aligned}
$$

At this coarse-grained level, there is again an exact equivalence between RTPs and ABPs upon identifying $\alpha$ and $(d-1) D_{r}$. This is particularly interesting since a uniform field can induce polar order [22], which thus does not by itself breaks the mapping between ABPs and RTPs.

Another way of breaking isotropy is to have the swimming parameters depend on the local orientation $\mathbf{u}$. For simplicity, we will only consider a first order spherical harmonic dependence $D_{r}(\mathbf{u})=D_{0}^{r}+\mathbf{D}_{1}^{r} \cdot \mathbf{u}$ and $\alpha(\mathbf{u})=$ $\alpha_{0}(\mathbf{u})+\boldsymbol{\alpha}_{1} \cdot \mathbf{u}$. We will also take $D_{t}=0$ for sake of clarity. Equation (2) now becomes

$$
\begin{gathered}
\dot{\psi}=-\nabla \cdot[v \mathbf{u} \psi]+\nabla_{\mathbf{u}} \cdot\left[\left(D_{0}^{r}+\mathbf{D}_{1}^{r} \cdot \mathbf{u}\right) \nabla_{\mathbf{u}} \psi\right] \\
-\left(\alpha_{0}+\boldsymbol{\alpha}_{1} \cdot \mathbf{u}\right) \psi+\alpha_{0} \varphi+\frac{1}{d} \boldsymbol{\alpha}_{1} \cdot \mathbf{p}
\end{gathered}
$$

Once again, we project this equation onto the three first harmonics. Tedious but straightforward algebra, that will be detailed elsewhere, show that equation (4) is not modified whereas $(56)$ become

$$
\begin{aligned}
\dot{p}_{i}= & -\nabla_{i}(v \varphi)-\left[\alpha_{0}+(d-1) D_{0}^{r}\right] p_{i}-\alpha_{1, i} \varphi \\
& -B_{i j k l}\left[\nabla_{j}\left(v Q_{k l}\right)+\alpha_{1, j} Q_{k l}+d D_{1, j}^{r} Q_{k l}\right]
\end{aligned}
$$

and

$$
\begin{aligned}
\frac{2 \dot{Q}_{i j}}{d+2}= & -B_{i j k \ell} \nabla_{k}\left[v p_{\ell}\right]-\frac{d}{\Omega} \int \mathrm{d} \Omega S_{i j k}^{(3)} S_{l m n}^{(3)} \nabla_{k}\left[v T_{l m n}\right] \\
& -\frac{2 \alpha_{0}+4 d D_{0}}{d+2} Q_{i j}-B_{i j k \ell}\left(\alpha_{1, k}+d D_{1, k}\right) p_{\ell} \\
& -\frac{d}{\Omega}\left[\alpha_{1, k}+2(d+1) D_{1, k}\right] T_{\ell m n} \int \mathrm{d} \Omega S_{i j k}^{(3)} S_{\ell m n}^{(3)}
\end{aligned}
$$

where $S_{i j k}^{(3)}=u_{i} u_{j} u_{k}-\left(u_{i} \delta_{j k}+u_{j} \delta_{i k}+u_{k} \delta_{i j}\right) /(d+2)$ is a tensor of 3rd order spherical harmonics and $T_{i j k}$ the component of $\psi$ along this tensor.

Equation (15) already shows that whatever the choice of coefficients, there can never be an equivalence between ABPs and RTPs in this context, since the tumbling yields a contribution $-\alpha_{1, i} \varphi$, which has no counterpart stemming from the rotational diffusion and which does not disappear in the large time/scale limit. Interestingly, the addition of a well chose angular potential for the rotational diffusion partially solves this problem. Such a potential produces an angular drift term $\dot{\psi} \sim-\nabla_{\mathbf{u}}\left(\mathbf{V}_{\mathbf{u}} \psi\right)$; choosing a drift $\mathbf{V}_{\mathbf{u}}=-\nabla_{\mathbf{u}} D_{r}$ then effectively replaces the term $\nabla_{\mathbf{u}}\left(D(\mathbf{u}) \nabla_{\mathbf{u}} \psi\right)$ in (4) by $\Delta_{\mathbf{u}}(D(\mathbf{u}) \psi)$, which adds a $-(d-1) D_{1, i} \varphi$ term to $(15)$. If this is done, the hydrodynamic equations for ABPs and RTPs do have similar structures, but it is still not possible to choose motility parameters to make all the coefficients match. Thus the mapping breaks down quantitatively. While these technical subtleties will be presented in more detail elsewhere, the results above are enough to prove that no general equivalence between ABPs and RTPs exists, once anisotropic dynamics is present.

Discussion. We comment finally on the role of the effective free energy $\sqrt{12}$ in relation to the concept of effective temperature. Several works have shown that active baths can be described by effective temperatures when dealing with passive tracers $[41-43$ or external potentials 14. 28, which implies that active particles may be considered as 'hot colloids'. For interacting ABPs, however, the usefulness of such effective temperatures has been questioned 16, 23. We have shown above that the phenomenology expected for interacting ABPs is fundamentally different from that of PBPs at any temperature. Nevertheless, under the specific conditions addressed here for which 12 is obeyed, equilibrium-like concepts do apply: the steady states of ABPs then satisfy detailed balance and so admit a free energy description. Currently we do not know the conditions (if any) under which a path can be traced from the microscopic dynamics of interacting $\mathrm{ABPs}$ directly to an effective free energy, avoiding the coarse graining steps that led us from (2) to (12). More generally, the validity or otherwise of effective temperature descriptions may largely depend on whether detailed balance emerges in a coarse grained description even though it is, by definition, absent microscopically in all active systems.

Conclusion. In summary, this Letter establishes that, under conditions where their local swim speeds and reorientation rates do not depend on the swimming direction, a large class of active particle models obey a common equation at the diffusion-drift level. The ABP and RTP limits are connected by a mapping, $\alpha \leftrightarrow(d-1) D_{r}$, between tumble rate $\alpha$ and rotational diffusivity $D_{r}$. Were tumbles to be considered of finite duration, one would expect a more complex mapping in which the swim speed $v$ of the equivalent ABPs also depends on $\alpha$ 7.

The diffusion-drift description treats all nonconserved density harmonics as quasi-stationary, and expands the total particle flux to first order in spatial gradients; it therefore requires slow variations in the conserved density (or zeroth harmonic). Since a description at this level is sufficient to explain the motility-induced phase separation of RTPs whose swim speed is density-dependent, ABPs will show such phase separations under exactly the same conditions on $v(\rho)$ as derived previously for RTPs. (In passing we have clarified that those conditions apply in $d=2,3$ as well as for $d=1$ as derived originally [7.) Motility-induced phase separation is caused by a feedback between (a) the tendency for particles to move slowly in regions of high density and (b) the tendency for particles to accumulate where they move slowly. The second of these is prohibited by detailed balance in thermal equilibrium. Our work shows that such phase separation (derived previously only for RTPs in 1D) is a robustly generic feature of interacting active particles. 
Furthermore, in cases where the dynamics is not isotropic, we have shown that the mapping can sometimes survive (even in the presence of polar order) but can also break down because of new couplings between harmonics of different orders. Nonetheless, progress might be possible when the angular dependencies on $\mathbf{u}$ are in turn expandable in low order angular or spherical harmonics as was the case in 32 .

Although the approach presented in this letter already can treat the sedimentation 14,22 and rectification 27 problems alluded to previously, direct interparticle forces remain to be explored. For example, interparticle attractions 25 44] or nematic interactions 21,33 promote clustering tendencies that seem distinct from the bulk phase separation caused by motility modulations alone. In general, interparticle forces create local orientational variation of the particle speed (just as the uniform force of sedimentation creates a global variation) 7 which effectively means $v=v(\mathbf{u})$ or $v=v(\mathbf{p})$. Accordingly, despite some initial steps that have been made recently [22 $29,30,32$ an explicit treatment of that case appears necessary as a first step towards a general dynamic density functional theory for interacting ABPs. Such a theory, which might involve analysis connected with that done for liquid crystals in [45], is a worthy goal for future research.

$$
* * *
$$

The authors thank Suzanne Fielding, Alasdair Thompson and Raphael Wittkowski for discussions. MEC thanks the Royal Society for a Research Professorship and KITP Santa Barbara for hospitality. JT thanks the Weizmann Institute of Science and the Technion Physics Department for hospitality. This work was supported in part by EPSRC Grant EP/J007404/1 and in part by the National Science Foundation under Grant No. NSF PHY11-25915.

\section{REFERENCES}

[1] Ramaswamy S., Annu. Rev. Cond. Mat. Phys., 1 (2010) 323.

[2] Cates M. E., Rep. Prog. Phys., 75 (2012) 042601.

[3] Romanczuk P. et al., Eur. Phys. J. Special-Topics, 202 (2012) 1.

[4] Schnitzer M. J., Phys. Rev. E, 48 (1993) 2553.

[5] Berg H. C., E. coli in Motion Springer, NY (2004).

[6] M. Polin et al., Science, 325 (2009) 487.

[7] Tailleur J. and Cates M. E., Phys. Rev. Lett., 100 (2008) 218103.

[8] Cates M. E. et al., Proc. Nat. Acad. Sci. USA, 107 (2010) 11715 .

[9] Liu C. et al., Science, 334 (2011) 6053.

[10] Fu X. et al, Phys. Rev. Lett., 108 (2012) 198102.

[11] Pacton W. F. et al., Ang. Chimie - Int. Ed., 45 (2006) 5420 .

[12] Howse J. R. et al., Phys. Rev. Lett., 99 (2007) 048102.

[13] Golestanian R., Liverpool T. B. and Ajdari A., New J. of Phys., 9 (2007) 126.
[14] Palacci J. et al., Phys. Rev. Lett., 105 (2010) 088304.

[15] Golestanian R., Phys. Rev. Lett., 108 (2012) 038303.

[16] Fily Y. and Marchetti M. C., Phys. Rev. Lett., 108 (2012) 235702.

[17] Redner G. S., Hagan M. F. and Baskaran A., arxiv: 1207.1737

[18] Thompson A et al., J Stat. Mech. Theo. Expt., (2011) P02029.

[19] Peruani F. and Morelli L. G., Phys. Rev. Lett., 99 (2007) 010602.

[20] Deseigne J., Dauchot O. and Chaté H., Phys. Rev. Lett., 105 (2010) 098001; Deseigne J. et al., Soft Matter, 8 (2012) 5629.

[21] Ginelli F. et al, Phys. Rev. Lett., 104 (2010) 184502.

[22] Enculescu M. and Stark H., Phys. Rev. Lett., 107 (2011) 058301.

[23] Bialké J., Speck T. and Löwen H., Phys. Rev. Lett., 108 (2012) 168301.

[24] Kaiser A., Wensink H. H. and Löwen H., Phys. Rev. Lett., 108 (2012) 268307.

[25] Theurkauff I. et al., Phys. Rev. Lett., 108 (2012) 268303.

[26] Fielding S. M., arXiv:1210.5464

[27] Galajda P. et al.,J. Bacteriol., 189 (2007) 8704.

[28] Tailleur J. and Cates M. E., EPL, 86 (2009) 60002.

[29] Bertin E., Droz M. and Gregoire G., J. Phys. A, 42 (2009) 445001.

[30] Mishra S. et al., Phys. Rev. E, 81 (2010) 061916.

[31] McCandlish S. R. et al., Soft Matt., 8 (2012) 2527.

[32] Farrell F.D.C. et al, Phys. Rev. Lett., 108 (2012) 248101.

[33] Peruani F., Deutsch A. and Bar M., Phys. Rev. E, 74 (2012) 030904.

[34] Llopis I. and Pagonabarraga I., EPL, 75 (2006) 999.

[35] Daintillan D. and Shelley M. J., Phys. Rev. Lett., 99 (2007) 058102.

[36] Sokolov A. el al, Phys. Rev. Lett., 98 (2007) 158102.

[37] Ishikawa T. and Pedley T. J., Phys. Rev. Lett., 100 (2008) 088103.

[38] Nash R. W. et al.,Phys. Rev. Lett., 104 (2012) 258101.

[39] In $2 \mathrm{~d}$, the angular harmonics are Fourier sine and cosine series in $\theta$; clearly p.u $=p_{1} \sin \theta+p_{2} \cos \theta$ and $\mathbf{Q}:\left(u_{i} u_{j}-\delta_{i j} / 2\right)$ span the subspaces generated by the two first harmonics and the two second harmonics, respectively. The same holds in $3 \mathrm{~d}$, where the components of any unit vector $u_{i}$ are linear combinations of $Y_{1}^{m}, m=$ $-1,0,1$ whereas the components of the traceless tensor $u_{i} u_{j}-\delta_{i j} / 3$ are linear combinations of $Y_{2}^{m}, m=-2 \ldots+2$. Note that $Q$ can be chosen traceless and symmetric.

[40] The derivation of Eq. 4. 6 rely on the fact that spherical and angular harmonics of order $\ell$ are eigenvectors of $\Delta_{\mathbf{u}}$ with eigenvalues $-\ell(\ell+1)$ and $-\ell^{2}$, respectively and on the equalities $\int u_{\alpha} u_{\beta}=\frac{\Omega}{d} \delta_{\alpha \beta}, \int u_{\alpha}^{4} \mathrm{~d} \Omega=\frac{3 \Omega}{d(d+2)}$ and $\int u_{\alpha}^{2} u_{\beta \neq \alpha}^{2} \mathrm{~d} \Omega=\frac{\Omega}{d(d+2)}$ where $\Omega$ is the total solid angle of the unit sphere.

[41] Wu XL. and Libchaber A., Phys. Rev. Lett., 84 (2000) 3017.

[42] Loi D., Mossa S. and Cugliandolo L. F., Phys. Rev. E, 77 (2008) 051111.

[43] Loi D., Mossa S. and Cugliandolo L. F., Soft Matt., 7 (2011) 3726.

[44] Schwarz-Linek J., et al, Proc. Nat. Acad. Sci. USA, 109 (2012) 4052. 
[45] Wittkowski R., Löwen H. and Brand H. R., Phys. Rev. E, 84 (2011) 041708. 